\title{
SYNTHÈSE DES CONCEPTS DE MODÉLISATION DU PHÉNOMĖNE DE MIGRATION DES CIVELLES D'ANGUILLA ANGUILLA (L., 1758) EN ESTUAIRE.
}

\author{
P. LAMBERT \\ CEMAGREF de Bordeaux, Division Aquaculture et Pêche, \\ 50 avenue de Verdun, BP 3, 33611 GAZINET Cedex, France.
}

\section{RÉSUMÉ}

Nous avons réalisé une synthèse bibliographique des différents modèles de migration des civelles d'anguille (Anguilla anguilla L.) en estuaire. Leur confrontation montre une convergence dans le choix des facteurs du modèle (température, débit, coefficient de marée). Par contre, des différences existent dans les types de relations (linéaires ou logistiques) qui lient ces paramètres aux captures par unité d'effort (CPUE) journalières issues de la pêcherie professionnelle. La modélisation du patron de migration n'est pas unique dans les différents modèles. Une réflexion sur la variabilité et sur la signification des CPUE semble indispensable pour améliorer la fiabilité des modèles. Des lacunes, en particulier la prise en compte de la variabilité des caractéristiques individuelles des civelles, sont constatées.

Mots-clés : anguille, Anguilla anguilla L., migration, estuaire, modélisation, facteurs de l'environnement.

\section{SYNTHESIS OF MODELISATION CONCEPTS OF MIGRATION PHENOMENA OF EEL CIVELLES (ANGUILLA ANGUILLA , L.1758) IN ESTUARY.}

\section{ABSTRACT}

We realized a review of the existent estuarine migration models for eel civelles (Anguilla anguilla L.). It shows that most of them focus on the factors involved in the models (temperature, waterflow, tide coefficient). But some differences exist in the relationships (linear or logistic) which link the parameters to the daily CPUE from the commercial fishery. Among the different models, there is not a single migration pattern for modelisation. It seems essential, in order to enhance the accuracy of the model, to engage a reflection on the variability and the significance of the CPUE. We noticed insufficient consideration to the individual variability of the eel civelle itself.

Key-words : eel, Anguilla anguilla L., migration, estuary, modelisation, environmental factors.

\section{INTRODUCTION}

Les données collectées à partir de la pêche commerciale dans les eaux intérieures européennes laissent supposer une diminution importante du stock d'anguille depuis le début des années 1980 (ANONYME, 1984 ; GUERAULT et al., 1986 ; GASCUEL, 1987 ; MORIARTY, 1987 ; BRUSLE, 1990 ; CASTELNAUD et al., 1994).

II importe donc, entre autres, pour tenter de dépasser ce constat, de comprendre et de quantifier les mécanismes de la migration estuarienne des civelles.

En reprenant la définition du terme civelle donnée par ELIE (1979) et ELIE et al. (1982), le phénomène migratoire étudié commence à l'arrivée des individus sur l'estuaire externe (zone marine sous influence estuarienne) jusqu'à la reprise d'alimentation en zone fluvio-estuarienne. 
Du fait du temps de transit relativement court des civelles dans l'estuaire dans des conditions hydrologiques normales (CANTRELLE, 1981), ce phénomène correspond pour la pêcherie commerciale au recrutement (arrivée sur les zones de pêche) et à la réforme (poissons devenant inaccessibles par la pêcherie pratiquant un métier donné). Par ailleurs, l'intensité de ce phénomène a de toute évidence une incidence sur le niveau de colonisation du bassin versant.

Sa compréhension est, par conséquent, la base de toute approche de dynamique de population à l'échelle du bassin versant et peut déboucher à terme sur la définition d'éléments d'aide à la décision dans le cadre de la gestion par hydrosystème des stocks de ce poisson.

L'objet du présent travail est de synthétiser les quelques tentatives de modélisation quantitatives des migrations estuariennes de civelles que nous avons répertoriées dans la littérature et de proposer des voies d'investigation pour des modélisations futures.

Nous ne retiendrons comme travaux que ceux ayant pour objet d'estimer à partir de facteurs du milieu (température, coefficient de marée, ...) les flux migratoires de civelles d'anguilles européennes dans un but de simuler soit une courbe observée (approche globale), soit les mécanismes de ce phénomène (approche déterministe).

Par ailleurs, les bases biologiques et écologiques de la migration font l'objet d'un article dans le présent ouvrage (ELIE et ROCHARD, 1994).

\section{LES DIFFÉRENTES TENTATIVES DE MODÉLISATION}

\section{Modèle DAVOUST et al. (1981)}

Ce travail concerne les migrations de civelles dans les estuaires de la Loire et de la Vilaine durant les saisons de pêche de 76-77 et 77-78. Les données de captures par unité d'effort (CPUE) sont obtenues pour chaque jour par le rapport entre les déclarations du lendemain des captures et le nombre de sorties.

Suite à une analyse spectrale des séries chronologiques, une relation linéaire multiple entre d'une part coefficient de marée et débit et d'autre part entre capture moyenne par sortie (lissée par une moyenne mobile de 3 jours) conclut ce travail :

$$
\text { CPUE }(j)=2+0.2 \mathrm{CM}(\mathrm{j})+0.0004 \mathrm{D}(\mathrm{j})
$$

avec $\operatorname{CPUE}(\mathrm{j})=$ capture par unité d'effort au jour $\mathrm{j}$.

$\mathrm{CM}(\mathrm{j}) \quad=$ coefficient de marée au jour $\mathrm{j}$.

$\mathrm{D}(\mathrm{j}) \quad=$ débit au jour $\mathrm{j}$.

Le coefficient de corrélation multiple pour ce modèle est de 0.34. A noter qu'une régression simple entre les captures moyennes par sortie et le débit ne modifie pas sensiblement la valeur du coefficient de corrélation (0.33).

\section{Modèle de BEN ABDALLAH (1991)}

Celui-ci est élaboré à partir des résultats de commercialisation des pêcheurs maritimes (au nombre de 40) et fluviaux (au nombre de 20 ) de l'estuaire de Loire pour cinq saisons (de 1984 à 1988).

Deux approches sont poursuivies :

- une analyse saisonnière : les cinq séries sont moyennées,

- une analyse inter-saisonnière : les cinq séries sont juxtaposées.

Pour chaque analyse, des composantes périodiques soit à 15 soit à 150 jours sont extraites. Puis sur chaque composante, des régressions avec retard, simples ou multiples, avec les paramètres explicatifs sont tentées. Enfin, des polynômes sont ajustés sur les composantes lissées. 
D'après cet auteur, il y aurait un triple effet des facteurs du milieu :

- un effet à court terme (entre 0 et 8 jours précédents) pour les coefficients de marée, la température et le débit,

- un effet à moyen terme (entre 8 et 20 jours) de la température,

- un effet à long terme (au delà de 20 jours) du débit.

Toutefois, les relations mises en évidence restent médiocres (10 à $40 \%$ de la variance des composantes saisonnières ou inter-saisonnières sont expliqués).

A noter que BEN ABDALLAH considère que les gradients de température et de débit n'ont pas d'impact sur les composantes lissées et des influences faibles sur les résidus.

\section{Modèle de ROCHARD (1992)}

Il est établi à partir des déclarations journalières de captures de civelles de pêcheurs échantillonneurs de l'estuaire de la Gironde durant les saisons 83-84, 85-86 et 87-88. (additives) :

De type descriptif, ce modèle décompose ce phénomène en deux composantes

- la tendance notée $T(t)$ représentée par une somme de trois gaussiennes,

- le rythme interne noté $F(t)$ et formalisé par une équation sinusoïdale, associée aux fluctuations du coefficient de marée (avec un à deux jours de retard).

L'équation de la tendance pour la saison $87-88$ est la suivante (le maximum de la première gaussienne est fixé arbitrairement à 90 jours pour permettre des comparaisons entre saisons) :

$$
T(t)=20.1 \exp \left(-.0014(t-90)^{2}\right)+7.9 \exp \left(-.0 .0013(t-150)^{2}\right)+3.6 \exp \left(-.001(t-120)^{2}\right)
$$

Le rythme interne peut être formalisé pour cette même saison par l'équation suivante :

$$
F(t)=12 \sin \left(\frac{\pi}{7.25} t+\pi\right)
$$

\section{Modèle de LAMBERT et al. (1994)}

Elaboré à partir du même type de données que dans le modèle de ROCHARD (1992) mais uniquement pour la saison $87-88$, ce modèle conceptuel présente une approche déterministe.

Il s'apparente à un modèle à compartiments (fig. 1) dont les flux entre les différents compartiments sont modélisés par des fonctions linéaires ou logistiques des paramètres du milieu (coefficient de marée, température de l'eau et débit fluvial) sauf pour l'arrivée des civelles sur le plateau continental, représentée par une gaussienne.

Le principe du modèle repose sur l'utilisation préférentielle du courant de flot par la civelle durant sa migration estuarienne (ELIE, 1979 ; GASCUEL, 1987 ; MACCLEAVE et WIPPELHAUSSER, 1987). Durant les phases de jusant ou lors de périodes défavorables (coup de froid, forte crue, ...), la civelle se plaque au fond de l'estuaire et ne remonte plus vers l'amont.

Les différents flux sont modélisés de la sorte:

$$
\begin{aligned}
f 1 & =\text { gaussienne }(h) \\
f 2 & =\text { linéaire }(C M(j-2))^{\star} \text { logistique }(T(t)) \\
f 3 & =\text { linéaire }(C M(j-2))^{\star} \text { logistique }(T(t))^{\star}(1-\text { logistique }(D(j)) \\
f 4 & =\left\{\begin{array}{l}
0 \text { si } h \neq \text { fin de journée } \\
1 \text { si } h=\text { fin de journée }
\end{array}\right. \\
f 5 & =\left\{\begin{array}{l}
f 2 \text { si } h=\text { début de journée } \\
0 \text { si } h \neq \text { début de journée }
\end{array}\right.
\end{aligned}
$$

avec $h=$ pas de programmation.

$j=$ jour. 


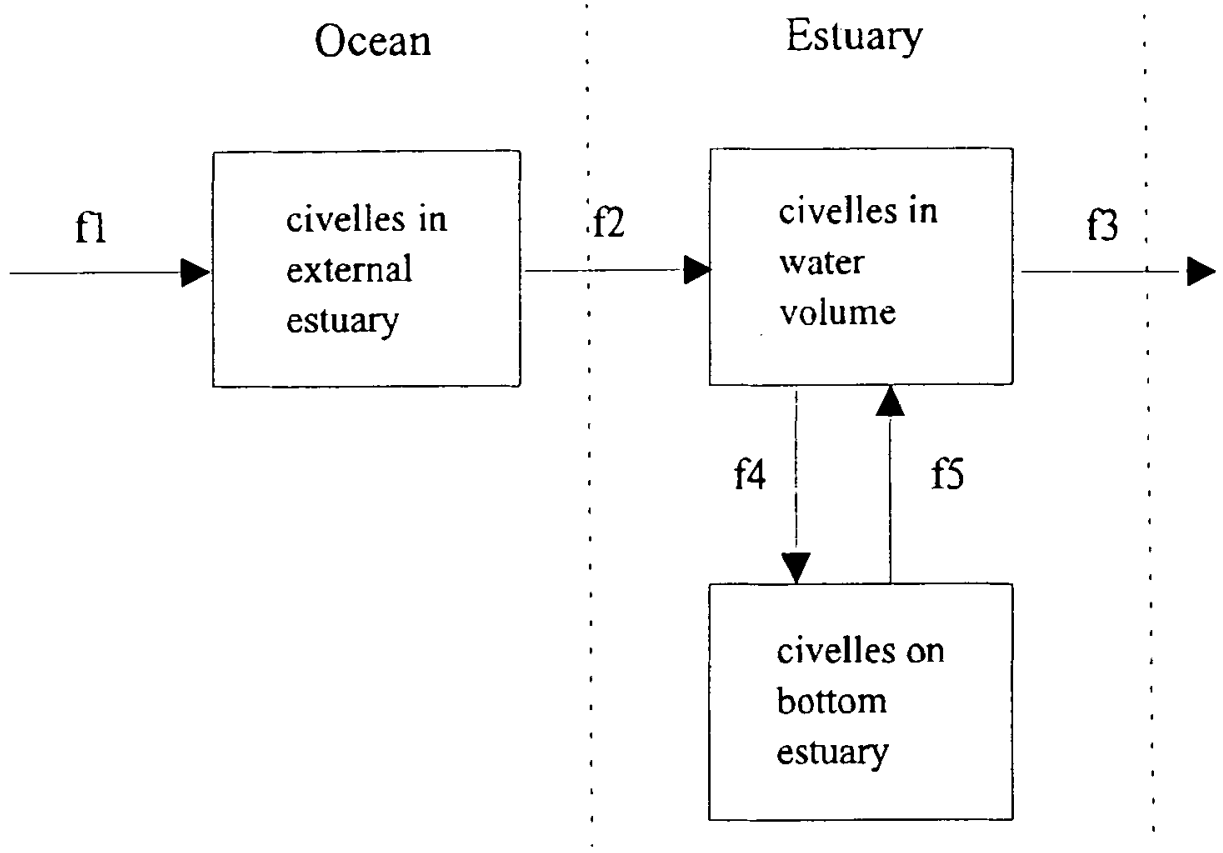

River

Figure 1 : Schéma conceptuel du modèle de LAMBERT et al. (1994).

Figure 1 : Conceptual diagram for the LAMBERT et al. model (1994).

De l'avis même de ses auteurs, ce modèle doit être amélioré en multipliant les deux compartiments estuariens et éventuellement en ayant recours à une modélisation individucentré pour tenir compte des variabilités individuelles.

\section{SYNTHÈSE ET DISCUSSION}

\section{La perception de phénomène migratoire}

Les données brutes de captures et d'effort

En zone estuarienne, les données sont toutes issues du «formidable outil d'échantillonnage des populations que constitue la pêche professionnelle" (CAILLART et MORIZE, 1991) et dont le rapport qualité-coût est meilleur que des pêches expérimentales sur des zones géographiques aussi vastes.

Par contre, elles présentent l'inconvénient de tronquer le phénomène à la période officielle d'ouverture de la pêche alors que le phénomène se déroule sur une partie plus importante de l'année, certes avec une intensité plus faible en dehors de cette période (ELIE et RIGAUD, 1984 ; GUERAULT et al., 1991 ; ELIE et ROCHARD, 1994).

Les contraintes importantes d'échantillonnage en zone estuarienne et la présence actuelle d'une pêcherie de civelles en France expliquent certainement que toutes les tentatives de modélisation de migration de civelles concernent des estuaires français.

Les données de captures sont obtenues soit auprès des mareyeurs (DAVOUST et al., 1981 ; BEN ABDALLAH, 1991) soit directement à partir des carnets de pêche que tiennent certains pêcheurs (ROCHARD, 1992 ; LAMBERT et al., 1994). Le seul critère de sélection des pêcheurs-échantillonneurs dans une zone considérée comme homogène est, chez certains auteurs, une assiduité minimum : $25 \%$ des jours de pêche pour chaque mois chez BEN ABDALLAH (1991) ou 40 jours par saison de pêche commençant le $1^{\text {er }}$ novembre pour se terminer le 15 avril pour LAMBERT et al. (1994). 
L'unité d'effort nominal (au sens halieutique du terme) est en général la journée de pêche d'un pêcheur (ROCHARD, 1992 ; LAMBERT et al., 1994) ou, dans certains cas, le nombre de sorties de pêche (ou, plus exactement, le nombre de livraisons) (DAVOUST et al., 1981).

Toutefois, aucun des auteurs des modèles élaborés à partir de ce type de données n'aborde la variabilité entre les pêcheurs. Pourtant, l'examen des moyennes des captures journalières des pêcheurs considérés comme assidus par LAMBERT et al. (1994) de l'estuaire de la Gironde montre pour la saison 1988 des différences dans un rapport de 1 à 10 (tableau I). Par conséquent, le choix de l'unité d'effort nominal (journée de pêche) semble masquer une grande disparité des puissances de pêche entre les pêcheurs (LAUREC et LEGUEN, 1981 ; GASCUEL, 1993 ; CASTELNAUD et al., 1994). Ces différences peuvent s'expliquer par des facteurs technologiques ou humains (GASCUEL, 1993). Les premiers correspondent à des armements non comparables, les seconds sont probablement liés à des temps de pêche effectifs différents ou à des stratégies de pêche plus ou moins adaptées...

A moins que le biais ne soit causé par l'hétérogénéité de l'abondance sur ce secteur de l'estuaire, déjà mis en évidence par CANTRELLE (1981) (augmentation d'aval en amont), probablement à relier avec les variations de largeur de l'estuaire.

Tableau I : Captures moyennes des différents pêcheurs de l'estuaire de la Gironde, ayant servi à établir la série chronologique de CPUE durant la saison 1987-1988 (données ayant servi dans LAMBERT et al., 1994).

Table I : Mean captures of the Gironde estuary fishermen, used to establish the CPUE time series during the 1987-1988 season (data used in LAMBERT et al., 1994).

\begin{tabular}{|c|c|c|c|c|c|}
\hline & $\begin{array}{c}\text { pêcheur } \\
1 \\
\text { (99 jours) }\end{array}$ & $\begin{array}{c}\text { pêcheur } \\
2 \\
\text { (65 jours) }\end{array}$ & $\begin{array}{c}\text { pêcheur } \\
3 \\
\text { (49 jours) }\end{array}$ & $\begin{array}{c}\text { pêcheur } \\
4 \\
\text { (53 jours) }\end{array}$ & $\begin{array}{c}\text { pêcheur } \\
5 \\
\text { (42 jours) }\end{array}$ \\
\hline moyenne sur la saison & 15.7 & 13.6 & 12.0 & 16.8 & 1.9 \\
\hline $\begin{array}{l}\text { moyenne sur } 11 \text { jours } \\
\text { de pêche communs }\end{array}$ & 25.3 & 14.8 & 15.5 & 24.8 & 1.9 \\
\hline
\end{tabular}

Enfin, les modèles sont tous élaborés à partir de données journalières, cette fréquence de mesure étant considérée comme la précision maximum réalisable.

Toutefois, en s'inspirant des fenêtres d'observation utilisées en analyse spectrale (LEGENDRE et LEGENDRE, 1984), cette fréquence n'est pas inférieure à la plus petite périodicité du phénomène écologique, le cycle circado-tidal. Ceci peut donc induire des erreurs d'interprétation et donc de modélisation. En effet, des phénomènes de repliement des spectres peuvent apparaître conduisant à la mise en évidence de périodicité qui n'existe pas.

\section{La signification des CPUE}

En première approximation, les CPUE (captures par unité d'effort), égales au rapport entre capture et effort, sont considérées comme des indicateurs de l'abondance (moyenne ou cumulée) sur une journée des civelles dans la masse d'eau (ELIE, 1979 ; LAUREC et LE GUEN, 1981 ; ROCHARD, 1992), l'abondance devant être comprise en terme de densité. 
Deux causes d'erreurs peuvent venir compliquer l'interprétation de la liaison entre abondance et CPUE :

- une abondance trop faible ; il y aurait, dans ce cas, relation non plus entre CPUE et abondance mais entre captures totales et abondance. Cette situation a été décrite par GASCUEL (1989) dans l'estuaire de la Loire ;

- une variabilité des puissances de pêche (confer supra).

Pour expliciter ce deuxième cas, supposons une abondance constante dans le temps, cinq pêcheurs dont les captures, négligeables par rapport au stock, sont strictement proportionnelles à l'abondance (donc constante dans le temps) mais dont les puissances de pêche présentent une forte disparité (tableau II). L'analyse des variables halieutiques classiques, captures, efforts, CPUE, suivant la grille de lecture de ROCHARD (1992), conduit alors à la conclusion d'une abondance variable, si les sorties des différents pêcheurs ne sont pas simultanées.

Tableau II : Exemple théorique de variations entre capture, effort, CPUE et abondance dans le cas d'une forte disparité entre pêcheurs.

Table II : Theoretical example of the variations between capture, effort, CPUE and abundance in case of strong disparity within fishermen.

\begin{tabular}{|c|c|c|c|c|c|c|c|c|c|}
\hline \multirow[t]{2}{*}{ Jours } & \multicolumn{5}{|c|}{$\begin{array}{l}\text { Captures des pêcheurs } \\
\text { (en kg) }\end{array}$} & \multirow{2}{*}{$\begin{array}{c}\begin{array}{c}\text { Captures } \\
\text { totales }\end{array} \\
\text { (en kg) }\end{array}$} & \multirow{2}{*}{$\begin{array}{c}\begin{array}{c}\text { Effort } \\
\text { total }\end{array} \\
(e n j)\end{array}$} & \multirow{2}{*}{$\begin{array}{l}\text { CPUE } \\
\text { (en kg/j) }\end{array}$} & \multirow[t]{2}{*}{ Abondance } \\
\hline & $n^{\circ} 1$ & $n^{\circ} 2$ & $n^{\circ} 3$ & $n^{\circ} 4$ & $n^{\circ} 5$ & & & & \\
\hline j & 1 & 7 & 7 & 10 & 10 & 35 & 5 & 7 & \\
\hline$j+1$ & & 7 & & 10 & 10 & $27(\Downarrow)$ & $3(\Downarrow)$ & $9(=)$ & $?$ \\
\hline$j+2$ & 1 & 7 & 7 & & 10 & $25(\Downarrow)$ & $4(\Uparrow)$ & $6.25(\Downarrow)$ & $(\Downarrow)$ \\
\hline$j+3$ & 1 & 7 & & 10 & & $18(\Downarrow)$ & $3(=)$ & $6(\Downarrow)$ & $(\Downarrow)$ \\
\hline$j+4$ & 1 & & 7 & & & $8(\Downarrow)$ & $2(\Downarrow)$ & $4(\Downarrow)$ & $(\Downarrow)$ \\
\hline$j+5$ & & & 7 & & 10 & $17(\Uparrow)$ & $2(=)$ & $8.5(\Uparrow)$ & $(\Uparrow)$ \\
\hline$j+6$ & & & 7 & 10 & 10 & $27(\Uparrow)$ & $3(\Uparrow)$ & $9(\Uparrow)$ & $(\Uparrow)$ \\
\hline$j+7$ & 1 & 7 & & 10 & 10 & $28(\Uparrow)$ & $4(\Uparrow)$ & $7(\downarrow)$ & $?$ \\
\hline
\end{tabular}

Cette difficulté peut être surmontée en standardisant l'effort de manière à approcher l'effort effectif (LAUREC et LE GUEN, 1981). De manière simple, on peut, par exemple, multiplier l'effort par le rapport, calculé sur les jours de pêche communs, entre la capture moyenne d'un échantillonneur pris comme standard et celle de l'échantillonneur considéré.

Cette correction n'est valide que sous l'hypothèse de la constance de la puissance de pêche au cours de la période étudiée (GASCUEL, 1993). Ceci paraît vraisemblable compte tenu de la faible durée de la saison de pêche, mais peut s'avérer inacceptable si le pêcheur adapte sa puissance de pêche en fonction de ses captures des jours précédents. En tout état de cause, l'acceptation de cette hypothèse pose un sérieux problème dans une analyse inter-annuelle. 
Par ailleurs, la définition d'un stock cryptique de civelles sur le fond de l'estuaire (LAMBERT et al., 1994) complique l'interprétation des CPUE (ou abondance) en terme de quantité d'animaux migrants puisqu'il n'y a plus de liens univoques entre ces deux notions : une augmentation de la CPUE peut être due soit à une forte arrivée, soit à un blocage des migrations amont, soit enfin à un «relargage» important des civelles à partir du fond de l'estuaire.

En poursuivant ce raisonnement, il ne peut y avoir de relations globales simples (linéaires) entre ces paramètres du milieu (température, débit, marée, ...) et les CPUE. En effet, un paramètre du milieu détermine (éventuellement) les flux entre les différents compartiments et donc, indirectement et de manière variable, l'abondance.

\section{Les paramètres explicatifs}

\section{Les paramètres du milieu}

Il est intéressant de noter la convergence dans le choix des variables du milieu considérées comme paramètres des modèles. II est évident que température de l'eau, coefficient de marée et débit fluvial sont (relativement) aisés à obtenir, il n'en reste pas moins vrai qu'une analyse bibliographique fait ressortir les variables thermiques et hydrodynamiques comme prépondérantes dans la dynamique du phénomène (ELIE et RIGAUD, 1984 ; LAMBERT et al., 1994 ; ELIE et ROCHARD, 1994).

Il est cohérent de constater que, d'une part, le débit a un effet positif en zone maritime (DAVOUST et al., 1981 ; BEN ABDALLAH, 1992) et négatif en zone fluviale (BEN ABDALLAH, 1992) et, d'autre part, qu'il est inhibiteur du flux amont dans le modèle de LAMBERT et al. (1994). Dans ce dernier cas, de forts débits bloquent la migration amont, ce qui a pour effet de diminuer l'abondance en zone fluviale au profit de la zone estuarienne.

Dans les modélisations récentes (BEN ABDALLAH, 1991 ; ROCHARD, 1992 ; LAMBERT et al., 1994), le coefficient de marée y est introduit avec un effet retard de 2 jours. Des effets retard avec d'autres paramètres sont explorés par BEN ABDALLAH (1991) et LAMBERT et al. (1994) mais rejetés dans ce dernier cas.

On peut toutefois regretter que dans le modèle de LAMBERT et al. (1994) débit et coefficient de marée ne sont utilisés que comme approximation de la vitesse du courant de flot (vecteur de la migration) sans que la nature des liens entre ces variables ne soit explicitée.

L'obtention des valeurs de température se fait pour le modèle de LAMBERT et al. (1994) à partir d'enregistrements au niveau d'une centrale nucléaire située à $60 \mathrm{~km}$ de l'embouchure de l'estuaire de la Gironde. Ce paramètre présente une variation spatiale en surface d'environ 1 degré (EDF thermographie) et le modèle thermique développé par GRAS et SABATON (1976) pour la partie médiane de cet estuaire garde une imprécision de l'ordre du degré.

Cette imprécision de mesure (ou de prévision par modèle) est en contradiction avec la sensibilité, de l'ordre de 2 degrés, du modèle de LAMBERT et al. (1994) vis-à-vis de la température. En effet, la courbe logistique de la température (donnée non publiée) présente une pente forte (phénomène de seuillage tout ou rien) : une température de $4{ }^{\circ} \mathrm{C}$ entraîne un blocage alors qu'une température de 6 degrés peut laisser passer le flux maximum.

\section{Les CPUE des jours précédents}

Ce type de modélisation fait appel aux modèles autorégressifs décrits par BOX et JENKINS (1970). II a été exploré par JELLYMAN et RYAN (1983) sur la migration d'anguillettes (Anguilla australis et $A$. dieffenbachii) dans le lac Ponui en Nouvelle-Zélande au travers de captures journalières dans une passe-piège.

L'inconvénient (a priori) de ces modèles est qu'il donne un poids important aux captures de la veille pour expliquer celles du jour, les paramètres environnementaux n'étant considérés que comme des "chocs" vis-à-vis du déroulement intrinsèque du phénomène (JELLYMAN et RYAN, 1983). II advient donc que l'essentiel de la migration n'est pas expliqué. 
Ces techniques doivent donc être réservées à la simulation (boîte noire) du phénomène. Toutefois, dans cette optique, ces méthodes testées sur les CPUE de l'estuaire de la Gironde donnent de bons résultats (LAMBERT, non publié).

\section{Les types de relation}

Les modèles DAVOUST et al. (1981), JELLYMAN et RYAN (1983) et BEN ABDALLAH (1991) reposent sur l'hypothèse de linéarité des relations entre captures et paramètres de l'environnement. L'acceptation de ce postulat découle probablement de la volonté de simuler plus que de chercher à élucider la dynamique du phénomène.

Une première amélioration est apportée par LAMBERT et al. (1994) en proposant des relations logistiques entre flux de civelles et paramètres environnementaux.

Toutefois, toutes ces relations restent monotones alors qu'il est probable, si l'on se réfère aux courbes de Shelford (SHELFORD et OLSON, 1937 in RAMADE, 1987) que l'on ait affaire à des courbes de préférendum en cloche. Ce type de courbe a été utilisé par LEGAULT et FEUNTEUN (1992) dans leur travail concernant la modélisation du recrutement fluvial d'anguillettes sur la Garonne. Ce modèle est établi à partir de captures au niveau d'une passe-piège sur le barrage de Golfech. Ils établissent une relation gaussienne entre le débit turbiné et le nombre d'anguilles capturées au niveau de la centrale par heure.

LAMBERT et al. (1994) ont montré qu'il existait une relation en cloche entre CPUE et température, mais considèrent que la partie décroissante de la relation n'est pas démontrée puisque les fortes températures ne sont observées qu'en fin de migration, période où le phénomène est moins intense.

\section{Le patron de migration}

Il est modélisé soit par une gaussienne (LAMBERT et al., 1994), soit par trois gaussiennes (ROCHARD, 1992), ou enfin par un polynôme (BEN ABDALLAH, 1991). Cette dernière solution a l'avantage de la dissymétrie mais l'inconvénient de ne pas autoriser des extrapolations avant et après la période d'observation, le polynôme donnant des valeurs rapidement trop aberrantes en dehors de la zone d'ajustement (valeurs négatives ne correspondant pas à l'observation in situ).

Dans le cas du modèle de LAMBERT et al. (1994), ce patron de migration est clairement explicité comme étant les arrivées sur le plateau continental. Chez ROCHARD (1992), il est associé à des vagues successives de pénétration dans l'estuaire.

Les polynômes ajustés sur les composantes lissées inter-saisonnières sont considérés par BEN ABDALLAH (1991) comme des indicateurs du recrutement au cours d'une saison de migration.

Dans le modèle de DAVOUST et al. (1981), il est indirectement pris en compte par la relation avec le débit : la tendance de ce paramètre du milieu suit grossièrement une parabole dont la concavité est tournée vers les valeurs positives et donc une liaison inversement proportionnelle permet de simuler le patron de migration. On retrouve ce même type de lien entre le patron de migration et un paramètre de milieu chez $B E N$ ABDALLAH (1991) qui estime que la composante lissée intra-saisonnière est surtout liée (de manière négative) avec la température.

Il est à noter la grande variabilité :

- du choix de la courbe représentant le patron de migration : courbe unimodale ou plurimodale, symétrique ou non,

- de l'interprétation qu'il en est fait : phénomène influencé par des processus marins (reproduction, migration transocéanique, ...) ou sous dépendance d'un ou de plusieurs paramètres du milieu continental (température, débit). 
Outre le choix du type de courbe, se pose le problème du déclenchement du phénomène migratoire, si l'on souhaite réaliser une modélisation sur plusieurs années. ROCHARD (1992) résout cette difficulté en calant arbitrairement le maximum de la première gaussienne à 90 jours. Les autres auteurs se réfèrent uniquement à la date calendaire, solution encore plus arbitraire.

\section{L'approche stochastique}

II est à regretter que pour l'instant les modèles actuels ne reposent pas sur des approches stochastiques pourtant plus en accord avec le contexte mathématique de séries chronologiques.

En effet, l'ensemble des tentatives de modélisation fait suite à une analyse d'une série écologique, encore appelée signal, qui se définit comme une longue suite d'observations ordonnées dans le temps (ou dans l'espace) (BOX et JENKINS, 1970 ; LEGENDRE et LEGENDRE,1979; LAUREC 1983). Par référence à BOX et JENKINS (1970) et à LAUREC (1984), une telle série doit être considérée comme une des manifestations possibles d'une loi commune. Mathématiquement, cette loi sera définie comme un processus aléatoire (c'est-à-dire suivant une loi probabiliste), le signal étant considéré comme une des réalisations ou trajectoires parmi celles que l'on aurait pu avoir, du processus dit "parental». Le signal constitue donc l'échantillon à partir duquel est pratiquée une inférence au processus.

A partir de l'hypothèse d'ergodicité, il est possible d'accéder aux propriétés du processus parental par la considération des moyennes sur un signal : l'observation sur une longue durée d'un signal permet de définir les lois probabilistes du processus parental à un instant donné.

Cette ambition confirme, avec une optique différente, la position de ROCHARD (1992). Cet auteur insiste en effet sur la nécessité, dans le cadre d'une mise en évidence d'évolutions, de stabilité dans le temps du réseau de pêcheurs-échantillonneurs même s'il n'est pas représentatif de la population de pêcheurs.

Par ailleurs, la variabilité comportementale de chaque individu (ELIE et ROCHARD, 1994) est a priori particulièrement grande, du fait de la variabilité des caractéristiques individuelles :

- stade de pigmentation induisant ou limitant une possibilité de transport diurne,

- poids éventuellement liés à des capacités d'utilisation du courant,

- sensibilité différente à la température entraînant des retards plus au moins grands dans le déplacement,

- activité thyroïdienne liée à des migrations, plus ou moins grande (FONTAINE, 1976).

Cette variabilité milite également en faveur d'une approche stochastique.

Enfin, ces différences peuvent être éventuellement accentuées par des phénomènes densité-dépendants comme la mortalité ou par des stimuli chimiques (ELIE et ROCHARD, 1994).

\section{CONCLUSIONS}

La modélisation des migrations de civelles a fait successivement appel à deux types de modèles :

- des modèles linéaires avec relations multiples entre d'une part CPUE et d'autre part débit, coefficient de marée, température et éventuellement la CPUE du jour précédent (DAVOUST et al., 1981 ; BEN ABDALLAH, 1991 ; ROCHARD, 1992), l'objectif étant de simuler le phénomène.

- un modèle déterministe à compartiments avec des flux simulés par des relations linéaires ou logistiques (LAMBERT et al., 1994), solution qui apparaît comme plus en accord avec les connaissances actuelles du phénomène (ELIE et ROCHARD, 1994). 
Une certaine convergence dans le choix des facteurs du modèle (température, coefficient de marée, débit) apparaît. Toutefois, compte tenu des problèmes de sensibilité des modèles par rapport aux variables physiques, un effort important sur le recueil, voire la modélisation de données de milieu (en particulier, la vitesse du courant de flot comme vecteur de migration et la température comme inhibiteur), devra être déployé pour espérer obtenir un modèle de migration de civelles opérationnel.

Le choix fait dans les différents modèles de la courbe représentative du patron de migration n'est pas unique. Pourtant cette étape qui définit a priori l'allure du phénomène est fondamentale dans la qualité du modèle. D'après la synthèse de ELIE et ROCHARD (1994), une gaussienne semble la plus appropriée pour simuler l'allure générale du phénomène de migration. Des expérimentations sur le plateau continental permettraient de conclure sur ce sujet.

Par ailleurs, l'unité de temps journalière pour l'observation des phénomènes, le calcul des CPUE à partir de pêcheurs dont l'efficacité n'est pas comparable, induisent des variations qui ne sont que des artefacts, dans les indices d'abondances utilisés pour caler tous les modèles. Pour pallier ces inconvénients, la modélisation devra à l'avenir respecter deux consignes minimales :

- le pas de temps pour la simulation devra être horaire (ou bi-horaire) pour tenir compte des variations des paramètres hydrophysiques de l'estuaire et de comportement des civelles liées au cycle de marée.

- l'ajustement du modèle se ferait par la comparaison du cumul journalier des quantités horaires de civelles dans la(es) masse(s) d'eau calculée(s) par le modèle avec la série des CPUE standardisées de l'ensemble de la pêcherie, voire avec les CPUE de chaque pêcheur.

II semble enfin intéressant d'avoir recours à une modélisation de type individu-centré (DEANGELIS et ROSE, 1992 ; LEPAGE, 1993) qui permettra de tenir compte au mieux à la fois de la variabilité individuelle des civelles et de la variabilité spatio-temporelle des paramètres hydro-physiques de l'estuaire. Dans ce cadre, les comportements individuels des civelles par rapport aux paramètres de l'environnement pourraient être simulés en faisant appel aux concepts de la logique floue. Ceci permettrait de garder toute souplesse dans la définition des relations entre mouvements des animaux et paramètres explicatifs (paramètre de l'environnement et caractéristiques individuelles) tout en y incluant la connaissance biologique sous forme linguistique.

Compte tenu de la prise en compte d'une activité humaine, ce modèle pourrait à terme être complété par des considérations sur la stratégie de pêche qui peuvent biaiser la perception du phénomène biologique au travers de la pêcherie.

Cette approche plus globale du système serait par ailleurs de nature à déboucher sur un réel outil de gestion de la population de civelles au travers des informations triées données par la pêcherie.

\section{BIBLIOGRAPHIE}

ANONYME, 1984. Rapport de synthèse et programme quinquennal. Groupe national anguille, $60 \mathrm{p}$.

BEN ABDALLAH L., 1991. Influence de quelques facteurs abiotiques sur l'abondance de civelle d'Anguilla anguilla dans l'estuaire de la Loire (France). CEPCl/FAO Working Party on Eel, Dublin (Irlande), Avril 1991, 12 p.

BOX G. E. P., JENKINS G. M., 1970. Time-series analysis, forecasting and control. New York, MacGraw-Hill. 575 p.

BRUSLE J., 1990. L'anguille européenne (Anguilla anguilla), une espèce jugée commune jusqu'à la décennie, mérite-t-elle d'acquérir aujourd'hui le statut d'espèce menacée? Bull. Soc. Zool. Fr., 114(3), 61-73. 
CAILLART B., MORIZE A., 1991. Potentiel halieutique d'un atoll de l'archipel des Tuamotu (Polynésie française) : méthodologie d'étude. In DURAND J.R., LEMOALLE J. et WEBER J, La recherche face la pêche artisanale, Symp. Int. Orstom-Ifremer, Montpellier, 1989.

CANTRELLE I., 1981. Etude de la migration et de la pêche des civelles Anguilla anguilla L. 1758 dans l'estuaire de la Gironde, Thèse de doctorat de 3ème cycle. CEMAGREF de Bordeaux, Div. A.L.A./ Université de Paris VI, 237 p.

CASTELNAUD G., GUERAULT D., DESAUNAY Y., ELIE P.,1994. Production et abondance de la civelle en France au début des années 90. Bull. Fr. Pêche Piscic., 335.

DAVOUST O., ELIE P., FONTENELLE G., 1981. Mise au point d'une méthode d'analyse des captures de civelles d'Anguilla anguilla $L$. dans les estuaires de la Loire et de la Vilaine. CIEM, CM 1981/ M : 34, 9 p.

DEANGELIS D.L., ROSE K.A., 1992. Which individual-based approach is most appropriate for a given problem ? In DEANGELIS D.L. and GROSS L.J., individuals-based models and approaches in ecology. Chapman \& Hall, 67-87.

ELIE P., 1979. Contribution à l'étude des montées de civelles d'Anguilla anguilla L., dans l'estuaire de la Loire : Pêche écophysiologie et élevage. Thèse de Doctorat de 3ème cycle, Université de Rennes I, 383 p.

ELIE P., LECOMTE-FINIGER R., CANTERELLE I., CHARLON N., 1982. Définition des limites des différents stades pigmentaires durant la phase civelle d'Anguilla anguilla $L$. (Poisson téléostéen anguilliforme). Vie Milieu, 32 (3), 149-157.

ELIE P., RIGAUD C., 1984. Etude de la population d'anguilles de l'estuaire et du bassin versant de la Vilaine (Pêche-Biologie-Ecologie). Examen particulier de l'impact du barrage d'Arzal sur la migration anadrome. Tome 1. Université de Rennes I/ CEMAGREF Div. ALA Bordeaux, $174 \mathrm{p}$.

ELIE P., ROCHARD E., 1994. Migration des civelles d'anguilles (Anguilla anguilla L.) dans les estuaires, modalités du phénomène et caractéristiques des individus. Bull. Fr. Pêche Piscic., 335.

FONTAINE M., 1976. Les mécanismes physiologiques des migrations des poissons. Oceanis, 2 (8), 343-363.

GASCUEL D., 1987. La civelle d'anguille dans l'estuaire de la Sèvre Niortaise, biologie, écologie, exploitation. ENSA Rennes. 4 (1), $353 \mathrm{p}$.

GASCUEL D., 1989. Seasonal dynamics of estuarine migration in glass eels (Anguilla anguilla, L.). Rapport ENSA de Rennes, $28 \mathrm{p}$.

GASCUEL D., 1993. Efforts et puissances de pêche : redéfinition des concepts et exemple d'utilisation. Communication au premier forum halieumétrique. Rennes, 1993. 18 p.

GRAS R., SABATON C., 1976. Etude du régime thermique de la Gironde. Rapport EDFdivision Echauffement et pollution des eaux et écologie.

GUERAULT D., BEILLOIS P., DESAUNAY Y., DOREL D., 1986. Variations of glass eel abundance from catch data : Loire and Vilaine regions. Vie Milieu, 36 (4), 237-241.

GUERAULT D., LECOMTE-FINIGER R., DESAUNAY Y., BIAGIANTI S., BEILLOIS P., GRELLIER P., 1991. The glass eels arrivals in the Vilaine estuary (Northern Bay of Biscay) through the year 1990 : demographic features and early life history. EIFAC Working Party on Eel, Dublin, Ireland, 28 p.

JELLYMAN D.J., RYAN C.M., 1983. Seasonal migration of elvers (Anguilla spp) into the lake Pounui, New Zealand, 1974-1978. New Zealand Journal of marine and freshwater research. Vol. 17, 1-15.

LAMBERT P., GRAMAGLIA A., ROCHARD E., ELIE P., 1994. Analysis of daily catch series and definition of a first model of civelles (Anguilla anguilla L., 1758) penetration in the lower estuary of Gironde (France). Communication au symposium international "Stock assessment in Inland Fisheries". Hull, 11-15 avril 1994. 
LAUREC A., 1984. Traitement des signaux quantitatifs et implications dans l'échantillonnage. In Frontier S. Stratégie d'échantillonnage en écologie. MassonPUQ. 217-270.

LAUREC A., LE GUEN J.C., 1981. Dynamique des populations marines exploitées. Tome 1, concepts et modèles. CNEXO Rapport scientifique et technique $n^{\circ} 45$. Brest, $117 p$.

LEGAULT A., FEUNTEUN E., 1992. Etude de la migration estivale des anguilles au barrage de Golfech sur la Garonne. Rapport FishPass/Migado, 25 p.

LEGENDRE L., LEGENDRE P., 1979. Ecologie numérique Tome 2, La structure des données écologiques. Masson, PUQ, Ed, $254 \mathrm{p}$.

LEGENDRE P., LEGENDRE L., 1984. Echantillonnage et traitement des données. In Frontier S. Stratégie d'échantillonnage en écologie. Masson-PUQ. 163-216.

LEPAGE C., 1993. Variabilité environnementale et structuration spatiale de la reproduction. Communication au premier forum halieumétrique. Rennes, $1993.15 p$.

MACCLEAVE J.D., WIPPELHAUSER G.S.,1987. Behavioural aspects of selective tidal stream transport in juvenile American eel (Anguilla rostrata). American Fisheries Society Symposium,1,138-150.

MORIARTY C., 1987. Factors influencing recruitment of the Atlantic species of anguillid eels. American Fisheries Society Symposium, 1, 483-491.

RAMADE F., 1987. Eléments d'écologie - écologie fondamentale. Mc Graw Hill. 369 p.

ROCHARD E., 1992. Mise au point d'une méthode de suivi de l'abondance des amphihalins dans le système fluvio-estuarien de la Gironde et application à l'étude écobiologique de l'esturgeon Acipenser sturio. Thèse de Doctorat de l'Université de Rennes I, $315 \mathrm{p}$. 